Artículo Original/Original Article

\section{El Síndrome de Burnout en Estudiantes de la Facultad Odontología de la Universidad Nacional de Córdoba}

\section{The Burnout Syndrome in Students of the Dentistry Faculty of the National University of Córdoba}

\author{
Casan E, Casan M, Ocampo M, Brochero G, \\ Ibañez C, Atala J L.
}

Universidad Nacional de Córdoba, Facultad de Odontología, Cátedra de Prostodoncia II A

\section{Resumen}

El Burnout, síndrome de estrés crónico, despersonalización y fatiga emocional. Los estudiantes logran desarrollar las competencias en su proceso formativo que favorecen el logro de sus objetivos académicos, mientras otros presentan dificultades que se registran en un elevado abandono académico, generalmente experimentan una alta carga de estrés durante su proceso formativo. Algunos desarrollan estrategias adecuadas para afrontar las exigencias académicas, otros no lo consiguen, y llegan a sentirse impedidos para modificar la situación problemática. Objetivo: analizar las causas determinantes, planteando estrategias para mejorar la labor docente asistencial. Método: se realizó un estudio transversal, descriptivo y analítico, a estudiantes de Prostodoncia II A, cohortes 2015 n=56 y 2016 n=74. Previo consentimiento aprobado por el CAIS, se formulo un cuestionario auto administrado y anónimo, con cinco preguntas generales, y propuestas por Maslach Burnout Inventory (M.B.I). Estadística y resultados: altamente positivos valoradas con tablas de contingencias y pruebas de Chi cuadrado. Las preguntas generales revelaron que el $87 \%$ presentaba cansancio por el ritmo de cursado, $58 \%$ le molestaba tener que realizar actividades extras, el $94 \%$ no aceptaba cambios inesperados sin previo aviso, el $89 \%$ por no contar con los recursos necesarios para el cursado y si quieren cambiar las condiciones de estudio actuales el $92.03 \%$. Con respecto (M.B.I) ,cuestionario que detecta la presencia de este síndrome y mide la frecuencia en tres dimensiones 1) Agotamiento Emocional (A.E):97.03\% y $87.05 \%$, 2) Despersonalización (D):98.6\% y 92.6\%, 3) Realización Personal (R.P):93.02\% y
83.9\%.Conclusiones los encuestados manifiestan un altísimo porcentaje de afectados por dicho síndrome, ya que para que éste se declare de forma fehaciente, los resultados deben encuadrarse en una escala predeterminada teniendo que revelar $\mathrm{AE}$ y $\mathrm{DP}$ altas $\mathrm{RP}$ baja. Por lo tanto se sugiere la creación de un departamento que atienda este tipo de problemática en forma continua y confidencial.

PALABRAS CLAVE: Sindrome Burnout, agotamiento, despersonalización.

\begin{abstract}
Introduction: Burnout syndrome - chronic stress, depersonalization exhaustion. Students are able to develop their skills in their training process, which foster the accomplishment of goals, other students present difficulties an elevated number of school leavers, who experience a high burden of stress during their training process. Some of them develop appropriate strategies to deal with the academic demands, and some of them don't, and they tend to feel themselves inadequate tochange their problematic situation. Objectives: To analyze the underlying causes by creating strategies toimprobe the supportive teaching work. Method: An analytical, descriptive, and cross-sectional study was carried out in students from the Department of Prosthodontics II A, cohortes 2015 n=56 and 2016 n=74. Previously consented and approved by CAIS, an anonymous, self-administered survey was conducted, consisting of five general questions and proposals by Maslach Burnout Inventory (M.B.I). Statistics and results: They were highly positive and they were obtained by using contingency tables and Chi-square test General revealed that 87 presented weariness due to the amount of hours in class, $58 \%$ were bothered by the fact that they had to do extra activities, 94\% did not accept unpredictable changes without previous notice, $89 \%$ stated that they do not have all the necessary resources for the classes and $92.03 \%$ think that current conditions for studying need to change. Concerning the M.B.I - the survey that shows the presence of this syndrome measures the frequency according to three dimensions: 1) Emotional exhaustion (A.E):97.03\% and 87.05\%,2) (D):98.6\% and 92.6\%,3 Personal fulfillment (R.P, Spanish acronym):93.02\% and 83.9\%.Conclusions: Respondents show a very high percentage of students affected by such syndrome, and for this to be declared credible, the results must be set in a pre-determined
\end{abstract}


scale, revealing elevated $\mathrm{AE}$ and $\mathrm{DP}$, and lower RP. Therefore, it is suggested the creation of an area where this kind of issue can be tackled continuously and confidentially

KEY WORDS: Burnout syndrome, exhaustion, depersonalization.

\section{Introducción}

Los estudiantes universitarios de carreras como la Odontología, especialmente en sus últimos años donde se atienden alumnos, se encuentran sometidos a un gran estrés, en muchos casos manifestando el síndrome de Burnout. Este hecho hace necesario analizar las diferentes causas a fin de elaborar estrategias a implementar para un mejoramiento en la metodología de enseñanza-aprendizaje, que permita alcanzar una mayor excelencia en la labor docente-asistencial, asumiendo nuestro compromiso para la formación de profesionales en competencias ${ }^{1}$. Estudios en docentes de la Facultad de Odontología como trabajo de tesis doctoral $^{2}$, el cual reveló gran incidencia de este síndrome. La bibliografía recopilada hace referencia que este síndrome se presenta y afecta con frecuencia a las personas que están en continuo trato con otras.

Los profesionales en los cuales se da con mayor incidencia este síndrome son los relacionados al área de la educación, salud, servicios sociales, entre otros ${ }^{3,4}$. Por lo tanto, es de gran relevancia estudiar y analizar el Síndrome de Burnout, en los estudiantes de cuarto año de Odontología, ya que se enfrentan a nuevas estrategia de aprendizaje, ya sea por el incremento de conocimientos en materias clínicas y por el comienzo casi a diario del tratamiento de pacientes, encontrándose los mismos dentro de esta franja de riesgo. La importancia de la realización del trabajo se basa en investigar en qué grado se ven afectados, ya que los mismos deben tratar con docentes, otros alumnos y pacientes y a su vez adquirir conocimientos para su formación profesional. Nos permitiría establecer el diagnóstico temprano de las alteraciones que pudieran presentarse, como así también iniciar la correcta derivación e intervención oportuna para revertirlas en el menor lapso de tiempo posible, teniendo en cuenta que es una problemática inédita que se podría analizar nuevamente en nuestra facultad considerando el grupo de estudiantes de cuarto año que se proponen en éste trabajo, estos generalmente experimentan una alta carga de estrés durante su proceso formativo. Algunos estudiantes logran desarrollar estrategias adecuadas para afrontar las exigencias académicas, mientras que otros no lo consiguen, y llegan a sentirse impedidos para modificar la situación problemática, lo cual trae como consecuencia el uso de comportamientos de escape o evitación como formas de afrontamiento que no son necesariamente las apropiadas en esta situación. Como se sabe, los problemas que no se resuelven crecen y se acompañan de un proceso acumulativo de malestar prolongado. Lo anterior puede contribuir a generar sensaciones de no poder dar más de sí mismo, tanto física como psíquicamente, una actitud negativa de crítica, desvalorización, pérdida del interés de la trascendencia, del valor frente al estudio y dudas crecientes acerca de la propia capacidad para realizarlo. El estudio del síndrome ha tenido un amplio desarrollo en los últimos años, y ha aportado a la comprensión de los procesos de estrés asistencial, organizacional y académico, con miras a mejorar la calidad de vida de los estudiantes.

El Síndrome Burnout (quemado, fundido) es un tipo de estrés laboral, un estado de agotamiento físico, emocional o mental que tiene consecuencias en la autoestima.

El estudio del Burnout en estudiantes universitarios es una línea investigación novedosa y muy reciente. Los estudios realizados en este sentido han estado dirigidos a la confirmación de la incidencia del síndrome en estas muestras pre profesionales. Las instituciones de educación superior suelen ser lugares altamente estresantes. En dichas instituciones, los alumnos se enfrentan de manera cotidiana a una serie de demandas 
(Docentes, Estudio, Compañeros, Pacientes) que bajo su propia valoración se constituyen en situaciones generadoras de estrés. Cuando son prolongadas y frecuentes, estas situaciones pueden conducir al síndrome de Burnout.

Por lo anteriormente expresado el objetivo de este trabajo fue evaluar la presencia de Síndrome del Quemado o Burnout en Estudiantes de la Facultad de Odontología de la Universidad Nacional de Córdoba para poder realizar un estudio Transversal Descriptivo y Analítico. Investigar la presencia del Síndrome de Burnout, en estudiantes que cursan cuarto año de la carrera de odontología. Identificar a este síndrome dentro del espectro de las patologías ligadas al trabajo y estudio.

\section{Métodos}

Se realizó un estudio transversal en estudiantes $(n=130)$, ambos sexos, de 4to año de la carrera de Odontología de la Universidad Nacional de Córdoba en los años 2015-2016, considerando separadamente los alumnos que cursaban el primero y segundo cuatrimestre.

Se aplicó un cuestionario autoadministrado de carácter anónimo validado (Maslach Burnout Inventory) $)^{5,6}$, que comprende 22 ítems relacionados a Despersonalización (DP), Realización Personal (RP).y de Agotamiento Emocional (AE) y otras variables como: cantidad de horas semanales que asisten a clases, edad, sexo, si posee algún trabajo.

\section{Análisis estadístico}

Las asociaciones entre las variables relevadas se evaluaron mediante tablas de contingencia y pruebas de Chi Cuadrado. Se fijó un p-valor < 0,05 para significación estadística.

El puntaje para cada constructo fue:

- Agotamiento emocional (Preguntas 1, 2, 3, $6,8,13,14,16,20)$. Puntaje total: 54 puntos (Categorías: Bajo: $\leq 13$; Medio: 14 a 23; Alto $\geq 24)$.
- Despersonalización (Preguntas 5, 10, 11, 15, 22). Puntaje total: 30 puntos (Categorías: Bajo: $\leq 2$; Medio: 3 a 8; Alto $\geq 9$ ).

- Realización personal (Preguntas 4, 7, 9, 12, 17, 18, 19, 21). Puntaje total: 48 puntos (Categorías: Bajo: $\leq 35$; Medio: 36 a 42; Alto $\geq 43$ ).

\section{Resultados:}

\section{Agotamiento Emocional}

Se observó un porcentaje de alto agotamiento emocional (puntuaciones mayores a 23), significativo en los alumnos que cursaban tanto el primero como el segundo cuatrimestre $(93,1 \%$ en promedio; $\mathrm{p}=0,029)$ (Fig. 1 ).

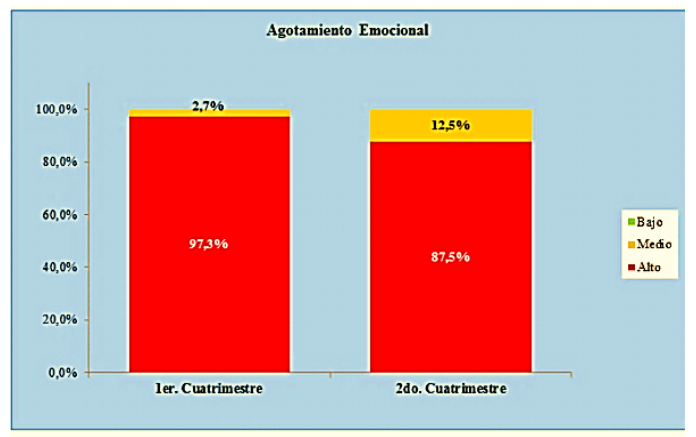

Figura 1. Nivel de agotamiento emocional

\section{Despersonalización}

Los porcentajes de alta despersonalización fueron muy elevados en ambos cuatrimestres, aunque disminuyó en el segundo cuatrimestre, de un $98,6 \%$ en el primer cuatrimestre al $92,9 \%$ en el segundo cuatrimestre, considerando la categoría de despersonalización alta (puntuaciones mayores a 8), aunque estas diferencias no resultaron significativas estadísticamente, $(\mathrm{p}=0,089)$ (Fig. 2). 


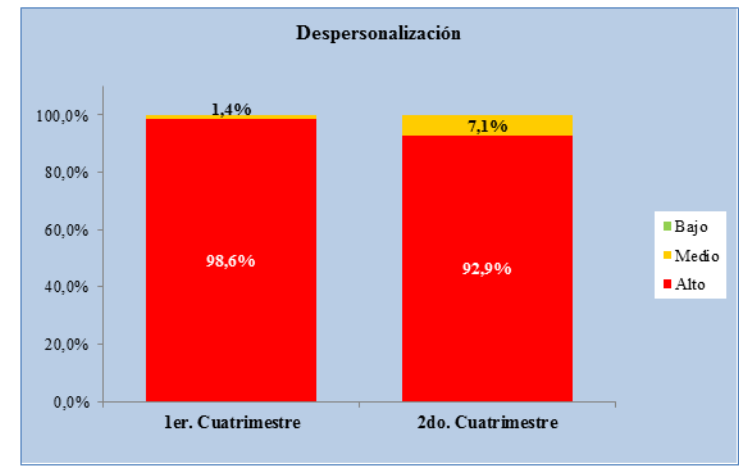

Figura 2. Nivel de despersonalización según cuatrimestre.

\section{Realización Personal}

Pese a que las diferencias de niveles de realización personal entre etapas no resultó significativa ( chi $^{2}$ de Pearson: valor $=4,092$; $\mathrm{p}>0,05), \quad \mathrm{Se}$ observaron porcentajes de realización personal bajos (puntuaciones menores a 36), que fueron menores en el segundo cuatrimestres $(83,9 \%)$, casi $10 \%$ menos que lo observado en la cohorte del primer cuatrimestre $(93,2 \%)$, no siendo significativo la diferencia entre los cuatrimestres $(\mathrm{p}=0,129)$ (Fig 3$)$.

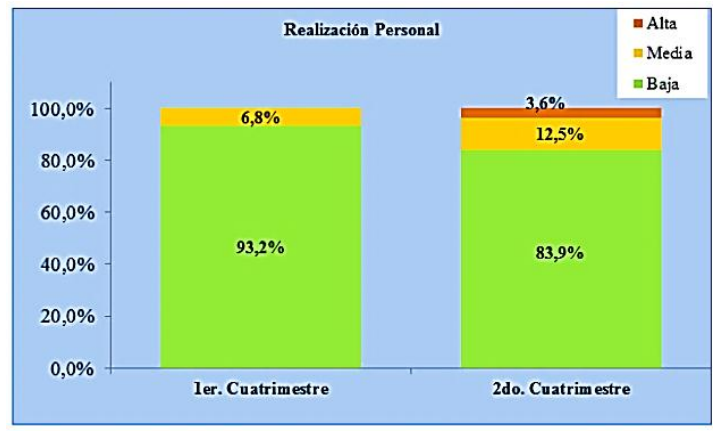

Figura 3. Nivel de realización personal según cuatrimestre.

\section{Discusión}

Nuestros resultados son coincidentes con el estudio realizado por Preciado-Serrano $\mathrm{M}$ y Vazquez-Goñi en estudiantes mexicanos de la Facultad de Odontologia pública donde los resultados determinaron que la conducta predictora del Sindrome de Burnout fueron las dimensiones de cansancio emocional y despersonalización y baja realización personal. Como así también encontraron porcentajes similares en relación al género y edad de los estudiantes afectados por el síndrome ${ }^{14}$.

Estudio de Burnout realizado por Martinez I y Pinto A en estudiantes universitarios de España, sus resultados revelaron que personas del sexo femenino han mostrado menos agotamiento y mayor eficacia académica por lo tanto hay menos tendencia a abandonar sus estudios ${ }^{15}$.

En estudios realizados por Picasso-Pozo, Nancy Huillca-Castillo N, y otros sobre Sindrome de Burnout en estudiantes de odontología de una universidad peruana, sobre 140 estudiantes, concluyeron El 30,7\% de la muestra presentó SB en grado severo, y el $23,6 \%$ en grado moderado. Se encontró que la prevalencia del SB era significativamente mayor en hombres, y menor en el grupo que vive solo. No se encontró relación entre el SB y el estado civil, trabajar además de estudiar, practicar alguna religión o deporte, o presentar molestias fisiológicas durante la época de estudios ${ }^{16}$.

Investigación de Misrachi-Launert et al. ${ }^{17}$ Fuentes de estrés percibidas y rendimiento académico de estudiantes de odontología chilenos sobre una muestra de 302 estudiantes concluyeron que el cuarto año parece ser el más estresante. El contacto temprano con los pacientes y una mejor planificación curricular y administración deben ponerse en práctica para evitar el aumento del estrés de la formación clínica ${ }^{17}$.

\section{Conclusión}

El estudio del Burnout en estudiantes universitarios es una línea de investigación novedosa han estado orientados a la confirmación de la incidencia del síndrome de Burnout donde los objetivos aportan resultados que pueden considerarse para la mejora del proceso enseñanza- aprendizaje. Se denota un aumento en la incidencia de este síndrome en el alumnado debido a la carga emocional, por el trato con pacientes $\mathrm{u}$ otras personas. 
Creemos que es necesario identificar a la población con una tendencia hacia el "desgaste", con el fin de establecer medidas preventivas y evitar el comportamiento futuro negativo en el trabajo, así como también a nivel personal. Por lo tanto se sugiere la creación de un departamento que atienda este tipo de problemática en forma continua y confidencial.

Todos los autores declaran que no existen conflictos potenciales de interés con respecto a la autoría y / o publicación de este artículo.

All authors declare no potential conflicts of interest with respect to the authorship and/or publication of this article

\section{Referencias}

1. UNESCO. Tecnologías de la información y comunicación en la formación docente- 2004 Disponible:

http://unesdoc.unesco.org/images/0012/001295/129 533s.pdf. Último acceso: 7-12-2017.

2. Casán E, Gómez de Ferraris ME, Rico R, Simbrón A. Identificación del síndrome del estrés en docentes de la Facultad de Odontología. Universidad Nacional de Córdoba, Argentina. Actual. Med. 2011, 96 (783): 38 -43.

3. Salvagioni DAJ, Melanda FN, Mesas AE, González AD, Gabani FL, Andrade SM. Physical, psychological and occupational consequences of job burnout: A systematic review of prospective studies. PLoS One. 2017;12(10):e0185781

4. Delgado C, Upton D, Ranse K, Furness T, Foster K Nurses' resilience and the emotional labour of nursing work: An integrative review of empirical literature. Int J Nurs Stud. 2017;70:71-88

5. Lastovkova A, Carder M, Rasmussen HM, Sjoberg L, de Groene GJ, Sauni R, Vevoda J, Vevodova S, Lasfargues $\quad G^{8}$, Svartengren $\quad M^{9}$, Varga M, Colosio C, Pelclova D. Burnout syndrome as an occupational disease in the European Union: an exploratory study. Ind Health. 2017, 3. doi: 10.2486/indhealth.2017-0132. .

6. Fumis RRL, Amarante GAJ, Nascimento AF, Vieira Junior JM. Moral distress and its contribution to the development of Burnout syndrome among critical care providers. Ann. Intensive Care. 2017; 7:71
7. Maslach C, Jackson SE, Leiter MP. MBI: Maslach Burnout Inventory Manual. 3rd ed. Palo Alto: Consulting Psychologists Press; 1996.

8. Maslach C, Schaufeli WB, Leiter MP. Job burnout. Annu Rev Psychol. 2001; 52: 397-422..

9. Characteristic of Staff Burnout in Mental Health Setting. Hosp.Community Psychiatry 1978. Apr. 29 (4) $233 / 7$.

10. Maslach C. Schaufeli. W. B. Leiter. M. P, "Job Burnout" Annu. Rev. Psychol, 2001.

11. Yépez Colmenares Germán. Modernización, medicina, enfermedades y salud pública en la ciudad de Caracas (1870-77). Hist. cienc. saudeManguinhos [Internet]. 2002 [cited 2017 Dec 11]; 9 (Suppl):89-109.Available from: http://www.scielo.br/scielo.php?script=sci_arttext\& pid $=$ S0104-

59702002000400005\&lng=en.http://dx.doi.org/10.1 590/S0104-59702002000400005.

12. Navarro-González D, Ayechu-Díaz A, HuarteLabiano I. [Prevalence of burnout syndrome and its associated factors in Primary Care staff]. Semergen. 2015; 41(4):191-8

13. Cherniss Cary (1980) "Stress in the Human Service".

14. Preciado-Serrano ML, Vázquez-Goñi JM. Perfil de estrés y síndrome de burnout en estudiantes mexicanos de odontología de una universidad pública. Rev Chil Neuro-Psiquiat 2010;48 (1): 1119.

15. Martínez IM, Marques Pinto MA. Burnout en estudiantes universitarios de España y Portugal y su relación con variables académicas. Universidade de Lisboa. Facultade de Psicología y Ciências da Educação. Alameda da Universidade. 1649-013. Lisboa, Portugal.

16. Picasso M, Huillca N, Ávalos J, Omori E, Gallardo A, Salas M. Síndrome de burnout en estudiantes de odontología de una universidad peruana. Kiru. 2012; 9(1):51-58. 24010-2717 (versión electrónica.

17. Clara Misrachi-Launert, Matías Ríos-Erazo, José M. Manríquez-Urbina, Cristián Burgos-Ibarra, Daniela Ponce-Espinoza. Fuentes de estrés percibidas y rendimiento académico de estudiantes de odontología chilenos. FEM: Revista de la Fundación Educación Médica versión On-line ISSN 20149840versión impresa ISSN 2014-9832

Correspondencia a/Corresponding to: Dr. Elías Casáns Correo electrónico/E-mail: elias.casan@unc.edu.ar 


\section{(c) (1) (9)}

